\title{
Le Congrès général 2019 de la SFP
}

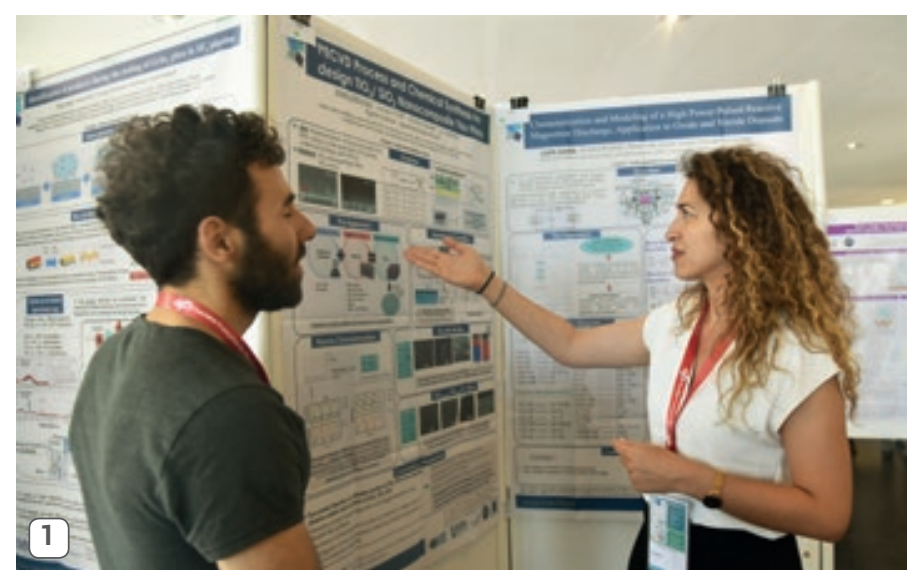

Conférences, sessions science et société, sessions posters, exposition industrielle (18 exposants), exposition photo, remises de prix ont rythmé la semaine. Un grand merci aux participants, conférenciers, exposants, bénévoles, qui ont contribué à ce que cette édition reste dans nos mémoires !

Treize conférences plénières, dont plusieurs proposées par des récipiendaires de prix prestigieux de la SFP et de ses partenaires internationaux, ont livré au public les derniers développements remarqués en physique (voir tableau 1). Les principaux domaines d'actualité ont été ensuite approfondis lors de vingt-trois sessions scientifiques spécialisées ("minicolloques") et de deux sessions par affiches (sessions «posters »).

\section{Les sessions posters}

Installées par les services de la Cité, elles étaient empreintes d'une atmosphère conviviale (fig. 1).

Des «prix posters " ont été attribués à trois doctorants, dont le jury a apprécié les résultats scientifiques, la clarté de la présentation et l'enthousiasme. De gauche à droite sur la figure 2 :

- Thomas Le Néel (Institut des Matériaux Jean Rouxel, Nantes), "Synthèse "verte" de nanoparticules d'or anisotropes pour spectroscopie Raman exaltée de surface et applications en biologie ".

- Laila Dakroub (Institut des Sciences Moléculaires d'Orsay), «Nouvel instrument pour la métrologie temporelle des sources XUV ultra-brèves".

- Ophélie Bugnon (Laboratoire Subatech, Nantes), «Mesure de l'excès de mésons $\mathrm{J} / \mathrm{Psi}$ à très basse impulsion transverse à $5 \mathrm{TeV}$, au LHC du CERN avec le détecteur ALICE ».

\section{Remise des prix SFP}

Cinq prix scientifiques de la SFP ont été remis durant le congrès. - Le prix Gentner-Kastler 2019 décerné - avec la DPG (Société allemande de physique) - à Christof Wetterich (Université d'Heidelberg), pour ses travaux théoriques sur la renormalisation fonctionnelle et les applications associées.

\section{Le $25^{\mathrm{e}}$ Congrès général de la Société Française} de Physique s'est déroulé du lundi 8 au vendredi 12 juillet 2019 à la Cité des congrès de Nantes, dans un cadre privilégié en centre-ville, à deux pas de la Loire et du château des Ducs de Bretagne. Organisé par un comité scientifique présidé par Jean Dalibard (Collège de France) et un comité local présidé par Jean-Luc Duvail (Institut des Matériaux Jean Rouxel), il a réuni 400 participants qui ont délivré 160 conférences et 66 communications par affiche.

- Le prix Friedel-Volterra 2018 décerné - avec la SIF (Société italienne de physique) - à Francesco Sette (ESRF, Grenoble) pour ses travaux pionniers sur le rayonnement X synchrotron. - Le prix Félix Robin 2017 à Danielle Dowek (ISMO, Orsay), pour ses travaux en physique atomique et moléculaire (voir Reflets de la physique $\left.\mathrm{n}^{\circ} 61, \mathrm{p} .41\right)$.

- Les prix de spécialité Aimé Cotton (physique atomique et moléculaire, optique) 2017 et 2018, respectivement à Patrice Genevet (CRHEA, Sophia-Antipolis) et Nicolas Sisourat (LCPMR, Paris 6) (voir pp. 34-35).

\section{Sessions science et société}

Sept sessions ont porté sur les nouveaux programmes de lycée, l'égalité-parité hommes-femmes dans la recherche, les publications (le plan national pour la science ouverte), l'histoire des sciences, l'emploi en entreprises des jeunes docteurs en physique, la désinformation scientifique, et les actions de coopération scientifique avec les pays en voie de développement. Elles ont été complétées par un atelier pratique d'écriture d'abstracts, organisé par EDP Sciences.

\section{Remise des prix du concours Beautiful Science}

Dans le cadre du congrès, la SFP a lancé Beautiful Science, un concours d'images et de sons sur le thème : "Montrer la science dans ce qu'elle a de plus beau et de plus élégant, de l'infiniment petit à l'infiniment grand! " (voir pp. 38-39). Une cinquantaine d'œuvres ont été exposées au Congrès général et les lauréats sont venus recevoir leur prix.

\section{Bilan parité/égalité du congrès}

Globalement, les femmes étaient bien représentées à ce congrès, principalement via les conférences plénières. En effet, ces dernières ont été données par sept femmes et sept hommes (bravo au comité scientifique et à son président !). 


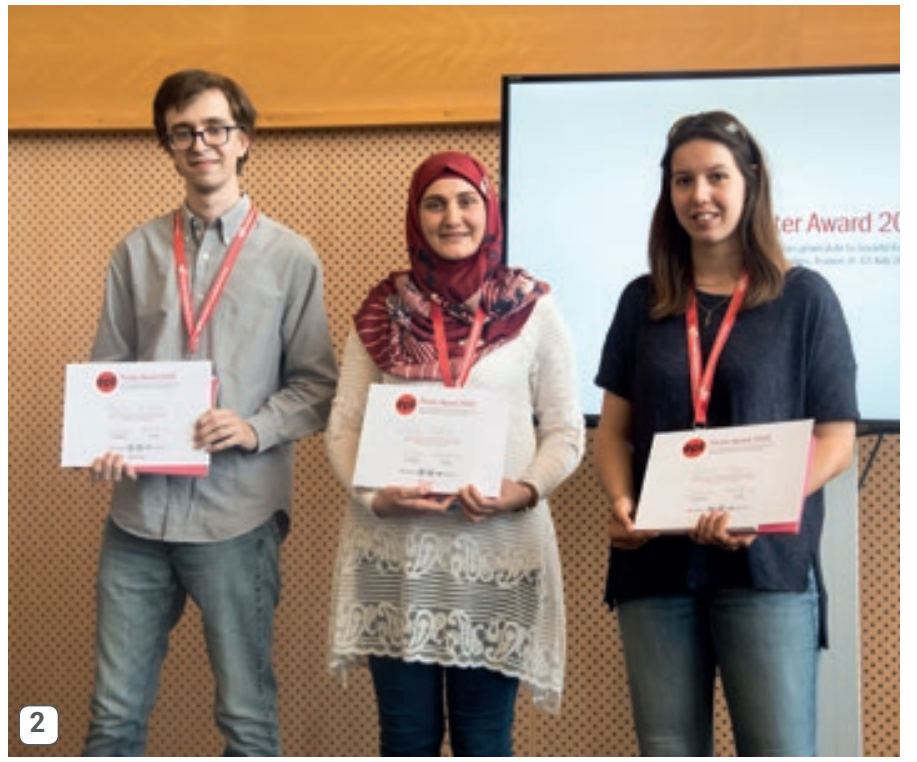

Le taux de femmes participant à la conférence était de $26 \%$, ce qui est légèrement supérieur au taux de femmes physiciennes en France $(\sim 22 \%)$, mais très supérieur à la proportion de participantes au congrès de Strasbourg en 2015 (12\%).

Sur l'ensemble des minicolloques, 33 présentations orales ont été données par des femmes, 108 par des hommes : donc 23\% de présentations par des femmes, taux inférieur à l'objectif de la charte de parité qui est, en physique, d'atteindre 30\%. On remarque une disparité entre les minicolloques, deux d'entre eux étant exclusivement masculins et deux autres totalement paritaires.

\section{Évènements grand public}

La SFP a organisé une conférence grand public, "La physique au service du patrimoine culturel : quand un accélérateur de particules perce les secrets des cuvres (d'art)! ", donnée par Claire Pacheco (fig. 3), responsable de l'Accélérateur Grand Louvre d'Analyse Élémentaire (AGLAE), Centre de recherche et de restauration des musées de France (C2RMF), Paris.

Trois visites de sites industriels ont été proposées le dernier jour : "Airbus Industries " (le site de production d'Airbus), "Technocampus Composites » et "Technocampus Océan » (deux plateformes de recherches technologiques mutualisées sur les matériaux et la robotique, dépendant de l'IRT Jules Verne).

\section{Photos et gazette}

Revivez quelques moments de la semaine au travers de la galerie de photos, disponible sur le site du congrès (www.sfp2019.fr).

Vous pouvez retrouver également sur ce site une vision décalée, toute en humour, de la semaine, en relisant les quatre numéros de la gazette Le Canard Intriqué, qui fut quotidiennement distribuée à la criée aux participants par notre joyeuse équipe de jeunes bénévoles.

Enfin, les organisateurs tiennent à remercier les nombreux partenaires qui ont soutenu le congrès avec enthousiasme.

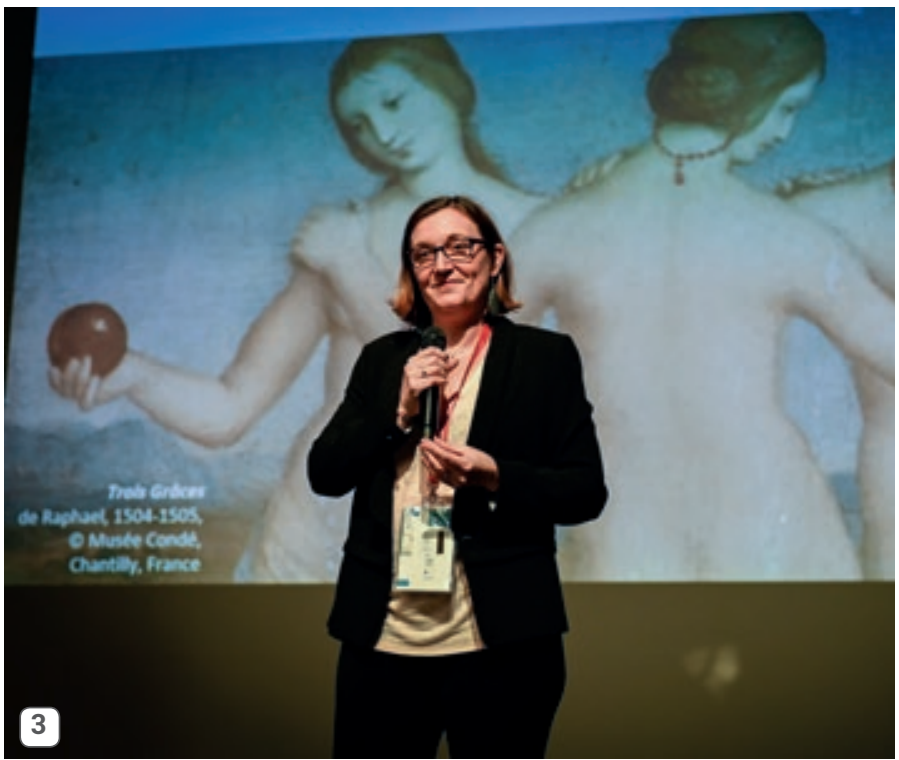

Liste des conférences plénières

- Les suspensions de particules actives, Jean-François Joanny (ESPCI et Institut Curie, Paris).

- Des réseaux de microcavités pour simuler la matière condensée avec de la lumière,

Jacqueline Bloch (C2N, Saclay/Polytechnique).

- Making the invisible, visible : the beauty of synchrotron science, Francesco Sette (ESRF, Grenoble).

- Functional renormalization from quantum gravity and cosmology to superconducting solids, Christof Wetterich ((Université de Heidelberg).

- Observer et contrôler les électrons pendant l'ionisation des molécules : du rayonnement synchrotron aux impulsions XUV attosecondes, Danièle Dowek (ISMO, Orsay).

- Physique de la turbulence à l'échelle de Kolmogorov, Bérangère Dubrulle (CEA/Saclay).

- Un plasma de quarks et de gluons est-il produit dans les collisions proton-proton au LHC?, Sarah Porteboeuf-Houssais (LPC, Clermont-Ferrand).

- Des masses des neutrinos à l'asymétrie matière-antimatière de I'Univers, Stéphane Lavignac (IPhT, Saclay).

- Nanocomposants pour le calcul inspiré du cerveau, Julie Grollier (UMR CNRS/Thalès, Palaiseau).

- Étudier le problème à N-corps avec quelques atomes, Antoine Browaeys (Institut d'Optique, Palaiseau).

- Structures et évolution de la Voie Lactée à la loupe avec la mission spatiale Gaia,

Annie Robin (Observatoire de Besançon).

- La physique des interfaces entre oxydes, Jean-Marc Triscone (Université de Genève).

- La fusion par confinement magnétique, Xavier Garbet (CEA Cadarache). 